\title{
Effects of intrathecal triamincinolone-acetonide treatment in MS patients with therapy-resistant spasticity
}

\author{
F Kamin ${ }^{1,2,7}$, PS Rommer ${ }^{1,3,7}$, M Abu-Mugheisib ${ }^{1,4}$, W Koehler ${ }^{5}$, F Hoffmann ${ }^{6}$, A Winkelmann ${ }^{1}$, R Benecke ${ }^{1}$ \\ and UK Zettl ${ }^{1}$
}

Objectives: Multiple sclerosis (MS) is an autoimmune disease affecting young people and is a major cause of disability. In the course of time, disability progresses and symptoms like spasticity may occur. Spasticity is a major cost factor in MS patients. Various agents are approved for the treatment of spasticity, but each of those agents may have several side effects. Intrathecally administered steroids (triamcinolone-acetonide (TCA)) may be efficient in treating spasticity in patients with lesions in the spinal cord and no response to first-line therapeutics. The aim of this study is to show effects of TCA treatment on clinical parameters in patients with MS.

Methods: This multicentre open label study included 54 patients with MS. The clinical outcome parameters were spasticity, disability, maximum walking distance, bladder function and quality of life. All patients received physiotherapy in addition to TCA treatment to obtain optimal effects on clinical parameters.

Results: Spasticity, maximum walking distance as well as disability improved significantly $(P \leqslant 0.001)$ during TCA applications. Bladder function improved in every seventh patient.

Conclusion: We observed the effects of intrathecally administered TCA on different clinical parameters including bladder function. TCA administration is a safe method to treat different symptoms in MS patients. Longitudinal trials with repeated TCA cycles are needed to show long-term effects. Besides TCA treatment, physiotherapy contributes to the improvement of clinical parameters.

Spinal Cord (2015) 53, 109-113; doi:10.1038/sc.2014.155; published online 16 September 2014

\section{INTRODUCTION}

Multiple sclerosis (MS) is an autoimmune disease affecting young people and is a major cause of disability. ${ }^{1}$ In the course of time, disability progresses and spasticity occurs in $>80 \%$ of MS patients. ${ }^{2}$ One-third of MS patients report spasticity that at least impact daily activities frequently. ${ }^{2}$ Besides spasticity, neurogenic detrusor overactivity is reported in MS patients with spinal manifestations and may lead to urinary incontinence. ${ }^{3}$ Treatment of spasticity includes physical therapy as well as therapeutic agents like baclofen and tizanidine. Recently, nabiximols (Sativex) has become available for treatment of spasticity in Europe. Each of these agents has a number of side effects. ${ }^{4}$ In patients with spasticity, high doses of baclofen or tizanidine may be needed and systemic side effects may occur. Nabiximols is a derivative of tetrahydrocannabinol and is subject to the narcotics law in respective countries. There is limited evidence that oral available antispastics like baclofen, diazepam, dantrolene and tizanidine are effective in the treatment of severe spasticity. ${ }^{5}$ Baclofen pumps have shown efficacy in the treatment of spasticity. However, invasiveness and higher costs have to be taken into account. Baclofen pumps may be restricted to patients with severe disabling spasticity. ${ }^{5}$ Another antispastic drug, tetrazepam, has recently been suspended across Europe after severe skin reactions have been observed including Stevens-Johnson syndrome, toxic epidermal necrolysis and drug rash with eosinophilia and systemic symptoms syndrome. ${ }^{6}$ Locally administered botolinum toxin may be used focally for muscle groups like hip adductors ${ }^{7}$ and its efficacy in treating neurogenic detrusor overactivity has been proven in phase III trials. ${ }^{3}$ Spasticity in MS patients represent an important cost factor for society. Direct non-medical costs are responsible for about two-thirds of all costs related with MS. ${ }^{8}$

Since the 1950s, glucocorticosteroids have been used intrathecally for prophylactic treatment of MS. Methylprednisolone-acetate led to an improvement of spasticity, but failed for prophylactic treatment. In addition, side effects like arachnoiditis occurred. In the past two decades, triamcinolone-acetonide (TCA) has been tested in small trials intrathecally and showed effects on expanded disability status scale (EDSS) and walking distance (EDSS decreased in the various trials between 0.5 and 0.7 , walking distance improved in one trial by almost $250 \mathrm{~m}) .{ }^{9-12}$ TCA doses range from 40 to $80 \mathrm{mg}$ every other day. Depending on the severity of spasticity, TCA is administered up to five times. Because of the pharmacokinetics of TCA, beneficial effects on spasticity may last up to 3 months. Mode of action of TCA is not completely understood and the scientific evidence of its efficacy relies on clinical observations. ${ }^{9-12}$ In vivo and in vitro experiments are also suggesting neurotoxic effects of glucocorticosteroids. ${ }^{13,14}$

The aim of this study is to show the effects of TCA treatment on clinical parameters like spasticity, EDSS, walking distance (mobility), quality of life (QoL) and bladder function in patients with MS.

${ }^{1}$ Department of Neurology, University of Rostock, Rostock, Germany; ${ }^{2}$ Department of Pathology, MVZ Hochstraße, Brandenburg an der Havel, Germany; ${ }^{3}$ Department of Neurology, Medical University of Vienna, Vienna, Austria; ${ }^{4}$ Department of Neurology, Klinikum Braunschweig, Braunschweig, Germany; ${ }^{5}$ Saechsisches Krankenhaus Hubertusburg, Wermsdorf, Germany and ${ }^{6}$ Staedtisches Krankenhaus Martha-Maria Halle-Doelau, Halle, Germany

${ }^{7}$ These authors contributed equally to this work.

Correspondence: Dr PS Rommer, Department of Neurology, University of Rostock, Gehlsheimer Straße 20, 18147 Rostock, Germany.

E-mail: stefan.rommer@med.uni-rostock.de

Received 3 March 2014; revised 10 July 2014; accepted 1 August 2014; published online 16 September 2014 
Table 1 Socio-demographic data of patients

\begin{tabular}{lccc}
\hline & Patients in total & Female & Male \\
\hline Number $(n)$ & 54 & 36 & 18 \\
Age (years) & $47.9 \pm 9.7$ & $48.7 \pm 9.7$ & $46.2 \pm 9.9$ \\
& & & \\
Course of disease & & & \\
$\quad$ rSPMS & 20 & 14 & 6 \\
SPMS & 26 & 19 & 7 \\
PPMS & 8 & 3 & 5 \\
Age at onset of disease (years) & $35.1 \pm 8.7$ & $35.5 \pm 8.8$ & $34.4 \pm 8.8$ \\
Disease duration (years) & $12.4 \pm 8.4$ & $13.0 \pm 8.5$ & $11.3 \pm 8.1$ \\
\hline
\end{tabular}

Abbreviations: PPMS, primary progressive MS; rSPMS, relapsing SPMS; SPMS, secondary progressive MS

Parameters were not normally distributed (Kolmogorov-Smirnov test).

\section{MATERIALS AND METHODS}

This multicentre open label study included 54 patients with MS according to the revised diagnostic criteria for MS. ${ }^{15}$ All patients entered a progressive phase, none of the patients reported relapses over the last months and did not receive glucocorticosteroid treatment. Concomitant antispastic treatment was allowed, but was not permitted to be changed during the study period. Magnetic resonance imaging of the brain and spine had to show no disease activity.

The trial was approved by the local ethics committee (University of Rostock) and has been registered with drks.de (DRKS00005671); all patients gave their written and informed consent prior to study entry.

Administration of TCA was performed on an in-patient basis by trained neurologists after exclusion of elevated intracranial pressure (done by magnetic resonance imaging). TCA was administered three up to five times every other day depending on the clinical judgement by the treating physician at doses ranging from 40 to $80 \mathrm{mg}$ (average dosage for patient with three TCA treatments was $127.4 \mathrm{mg}$ s.d. \pm 22.3 and $205.9 \mathrm{mg}$ s.d. \pm 37.3 for five administrations) every other day. In all cases atraumatic 'Sprotte' cannulas (Pajunk Medizintechnologie GmbH, Geisingen, Germany) were used for lumbar puncture. TCA suspension dissolved in saline was administered.

Clinical assessments were done before the first administration, after TCA treatment and at discharge. Spasticity was evaluated on Ashworth scale; QoL and bladder function were assessed on self-reporting scales; walking distance was evaluated by physiotherapists.

Each patient received functional physiotherapy adapted to the individual requirements (spasticity, weakness and ataxia). In each patient, muscle stretching, standing exercises, gait training and relaxation exercises were performed. Patients received one unit of physiotherapy a day (60 min).

\section{Statistical analyses}

Statistical analyses were performed with software SPSS 15.0 (Chicago, IL, USA) and IBM SPSS Statistics version 20 (Armonk, NY, USA). KolmogorovSmirnov test was used for checking distribution in all parameters. Sociodemographic and clinical parameters were analysed with the Kruskal-Wallis one-way analysis of variance and Mann-Whitney $U$-test $(P=0.05)$

\section{RESULTS}

Fifty-four patients were included in the trial, of which two-thirds were female. All patients entered a progressive phase, some of them had relapses within the last year-relapsing secondary progressive MS. Mean age was 47.9 years and baseline EDSS was 5.8. The patient groups did not differ between male and female regarding the different parameters. See Tables 1 and 2. All patients received physiotherapy in addition to TCA treatment.
Table 2 Baseline characteristics of clinical parameters

\begin{tabular}{lcccc}
\hline $\begin{array}{l}\text { Clinical } \\
\text { parameters }\end{array}$ & $\begin{array}{c}\text { Patients in } \\
\text { total (average } \\
\text { at baseline }\end{array}$ & $\begin{array}{c}\text { Female } \\
\text { (average } \\
\text { and s.d.) }\end{array}$ & $\begin{array}{c}\text { Male } \\
\text { (average } \\
\text { and s.d.) }\end{array}$ & $\begin{array}{c}\text { Comparison } \\
\text { between } \\
\text { groups }\end{array}$ \\
\hline EDSS & $5.8 \pm 1.6$ & $5.7 \pm 1.7$ & $6.2 . \pm 1.4$ & n.s. \\
$\begin{array}{l}\text { Ashworth scale } \\
\text { Walking }\end{array}$ & $2.2 \pm 1.3$ & $2.1 \pm 1.3$ & $2.4 \pm 1.4$ & n.s. \\
$\begin{array}{l}\text { distance (m) } \\
\text { Quality of life }\end{array}$ & $4.6 \pm 1.9$ & $4.8 \pm 2.1$ & $4.3 \pm 1.6$ & n.s. \\
\hline $\begin{array}{l}\text { Abbreviation: EDSS, expanded disability status scale. } \\
\text { Parameters were not normally distributed (Kolmogorov-Smirnov test). Patient groups did not } \\
\text { differ between female and male (Mann-Whitney U-test). }\end{array}$ & & & \\
\end{tabular}

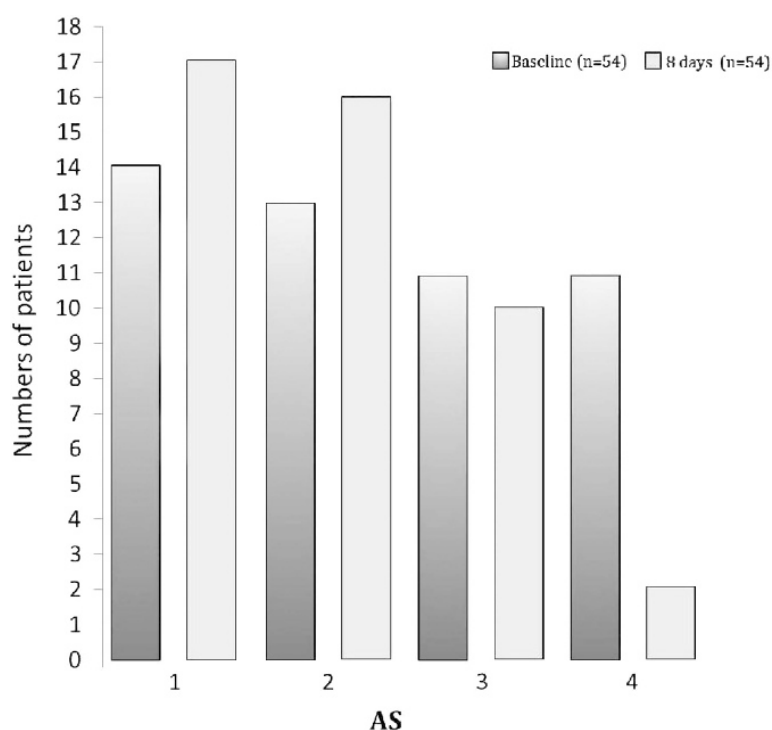

Figure 1 Change of the Ashworth scale during first cycle of TCA treatment. After treatment with TCA the proportion of patients with low spasticity (Ashworth scale 1 or 2) was increased significantly.

\section{Ashworth scale}

Spasticity was measured on Ashworth scale and improved significantly $(P \leqslant 0.001)$ during TCA treatment and physical therapy through all patient subgroups. See Figure 1. Baseline Ashworth scale value in all patients was 2.2 (s.d. \pm 1.3 ) and improved to 1.6 (s.d. \pm 1.1 ). Whereas baseline spasticity was higher in male patients (2.4 (s.d. \pm 1.4$)$ vs 2.1 (s.d. \pm 1.3$)$ ), treatment led to a similar effect for both gender groups. The improvement did not differ significantly between three and five TCA applications.

\section{EDSS}

EDSS was reduced significantly during TCA treatment from 5.8 (s.d. \pm 1.6$)$ to 5.5 (s.d. \pm 1.7$)(P \leqslant 0.001)$. See Figure 2 . The effect was shown in both genders. The effect was stronger in male patients.

\section{Maximum walking distance}

Walking distance improved significantly $(P \leqslant 0.001)$ during treatment on average in all patients from $247 \mathrm{~m} \mathrm{(s.d.} \mathrm{\pm 302)} \mathrm{to} 322 \mathrm{~m}$ (s.d. $\pm 346 \mathrm{~m}$ ). Time for assessment of maximum walking distance was not limited. 


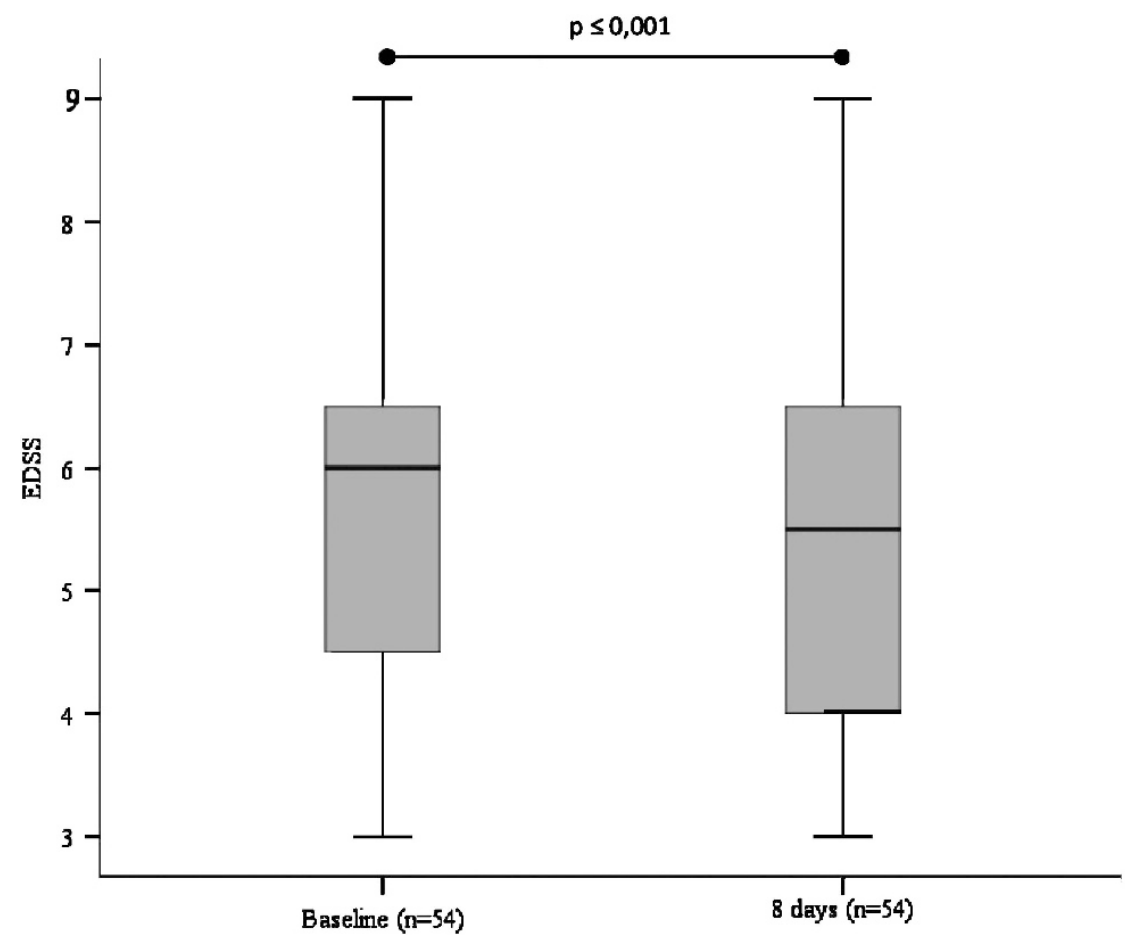

Figure 2 Change of the EDSS in the first cycle of TCA treatment. Statistical analysis: Friedman test and Wilcoxon test. $n$, number of patients.

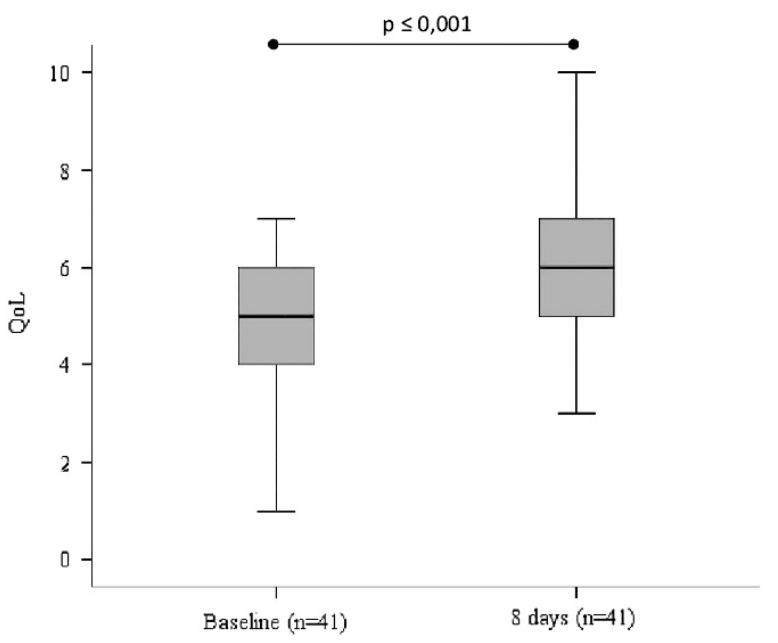

Figure 3 Changes in the QoL during the first cycle of TCA treatment. Statistical analysis: Friedman test and Wilcoxon test. $n$, number of patients.

\section{Bladder function}

Patients had to classify their urinary function into 'continent', 'incontinent' and 'feeling of residual urine'. After TCA treatment, bladder function was assessed again according to the abovementioned criteria. In addition, an improvement could be indicated by the patients. At baseline, 31 patients reported continence, whereas 21 patients reported incontinence or feeling of residual urine. After TCA treatment, 18 patients reported incontinence or feeling of residual urine, whereas 32 patients reported continence and 2 patients reported an improvement of their urinary function.

\section{Quality of life}

TCA application led to higher QoL. QoL was assessed semiquantitatively on an analogue scale (values from 0 (poor) to 10 (very good)). QoL improved significantly from 4.6 (s.d. \pm 1.9 ) to 5.7 (s.d. \pm 1.7 ) at the end of the cycle $(P \leqslant 0,001)$. See Figure 3 . The effects were more pronounced in male patients, but did not differ significantly between the genders.

\section{DISCUSSION}

We report on intrathecally administration of TCA in 54 MS patients with severe spasticity with no response to first-line therapeutics.

Our trial is one of the largest prospective studies for TCA treatment in MS patients, and results are in conformity with published trials showing that TCA intrathecally administered led to an improvement of spasticity in MS patients. ${ }^{11,12}$ However, in contrast to former trials, we have not only evaluated effects on spasticity and EDSS, but also changes in walking distance, bladder function and QoL were looked at. Intrathecally applied TCA led to a significant improvement in all clinical parameters during treatment.

Patients in our trial suffered from moderate spasticity on average (2.2 points on Ashworth scale). TCA treatment led to an improvement by 0.6 points. Although this improvement seems to be minor, it may lead to an extension of the walking distance in two-third of the patients, whereas only four patients $(7.5 \%)$ worsened on walking distance. Walking distance increased on average by 30\%. Both parameters (decreased spasticity and extended walking distance) may indicate reduced disability. EDSS decreased in one-third of the included patients. In addition, self-reporting of bladder function showed an improvement in every seventh patient. Reduced spasticity will enable patients to be more mobile; spasticity is a disagreeable sensation and often painful. It is often associated with depression. ${ }^{16,17}$ An improvement of $50-100 \mathrm{~m}$ in walking distance enables the patient to gain control over more daily activities. Together these 
improvements led to an improvement of QoL by 1.3 points on the 10point rating scale. Interestingly, these results were observed through all patient groups (relapsing and non-relapsing secondary progressive MS,primary progressive MS, male and female patients). Effects did not differ significantly whether TCA applications were performed three or five times.

No severe side effects were observed in our study (back pain and headache were treated with analgesics). None of the patients was hindered from participating in physiotherapy. No infections were detected, no arachnoiditis was reported. There were no signs of aseptic meningitis. Common side effects were headache in up to every tenth patient. Cell count in the CSF was 10.8 cells $\mu \mathrm{l}^{-1}$ (s.d. 8.2) on average after three application and 6.3 cells $\mu l^{-1}$ (s.d. 6.2) after five applications. Highest cell count was 64 cells $\mu l^{-1}$. None of the patients showed symptoms for infection. No severe side effects occurred. The small number of side effects may be drawn back to the use of atraumatic needles and were in the range of published trials. ${ }^{11,12}$

Intrathecal TCA treatment seems to be a safe and an effective option to treat moderate spasticity in patients with no response to first-line antispastic agents.

Our study is of relevance in times of scarce resources. According to a recent study, spasticity is responsible for about $€ 115000$ a year per patient. Interestingly, the costs for severe spasticity were 2.4 times higher than that for mild spasticity. ${ }^{8}$ Besides economic factors, spasticity is a major cause of disease burden in MS patients, ${ }^{2}$ which even may lead to muscle rupture. ${ }^{18}$

We are aware of limitations to our results. Physical therapy will also lead to improvement of walking distance and spasticity, and will influence EDSS. However, for achieving optimum benefit to spasticity in patients, TCA treatment should be performed accompanied by regular physical therapy. ${ }^{19}$ Personal care will lead to improvement of QoL. QoL and bladder function were self-assessed by the patients, which may lead to subjective results. Nevertheless, QoL measures on a semi-quantitatively scale have shown to be suitable for outcome measure. $^{20}$

A placebo-controlled trial is ethically not possible because of the invasiveness of lumbar puncture.

Furthermore, as this was a multicenter trial, there have been several investigators that may lead to individual clinical judgment on severity and necessity of TCA administrations. Nevertheless, on the other side, each of the investigators is specialized on treatment of MS patients.

\section{CONCLUSION}

Intrathecal TCA treatment is a safe way of treating spasticity. Repeated TCA administrations are an alternative of treating therapy-resistant spasticity in select patients. Invasiveness of lumbar puncture might limit the usage of TCA administration.

This study emphasises the positive impact of TCA treatment on the different outcome functions in MS patients. In addition, bladder function was assessed. It represents a common problem in MS patients that has not gained a lot of awareness. The result of bladder function has to be confirmed by cystomanometry. Long-term results are necessary to see sustained effects on different clinical parameters.

Whereas TCA treatment seems to be an appropriate mean of treating moderate spasticity in patients with no sufficient response to first-line therapeutics, baclofen pumps may be an option for patients with severe spasticity.

\section{DATA ARCHIVING}

There were no data to deposit.

\section{CONFLICT OF INTEREST}

PSR served as consultant to Bayer Pharma AG and received speaker honorary from Shire. He received travel support from Biogen Idec. He has recently entered employment with Novartis. WK received travel support, research grant and speaker fees from Biogen Idec, Bayer Healthcare, Merck-Serono, Novartis and Teva. FH received travel support from Bayer Healthcare and Biogen Idec; received research grants from Allergan, Bayer Healthcare, Biogen Idec, Genzyme, Ipsen, Merz, Novartis, Teva and UCB; received speaker or consultant fees from Allergan, Bayer Healthcare, Biogen Idec, Genzyme, Ipsen, Merz, Novartis and Teva. AW received travel support from Novartis and Genzyme; received speaker and consultant fees from Merck-Serono and Genzyme. UKZ received speaker fees from Almirall,Bayer Healthcare, Biogen Idec, Genzyme, Merck-Serono, Novartis, Sanofi and Teva. The remaining authors declare no conflict of interest.

\section{ACKNOWLEDGEMENTS}

We thank Ms Nele Gillwaldt for providing figures.

Author contributions: FK contributed to the design and conceptualisation of the study, analysis and interpretation of the data, conducted the statistical analysis, and revised the manuscript. PSR contributed to the design and conceptualisation of the study, analysis and interpretation of the data, drafted the manuscript, and revised the manuscript. FK and PSR contributed equally to this research paper, and are both listed as first authors. MAM WK $\mathrm{FH}, \mathrm{AW}$ and RB contributed to the analysis and interpretation of the data, and revising the manuscript. UKZ contributed to the design and conceptualisation of the study, analysis and interpretation of the data, and revising the manuscript.

1 Weinshenker BG. The natural history of multiple sclerosis: update 1998. Semin Neurol 1998; 18: 301-307.

2 Zwibel HL. Contribution of impaired mobility and general symptoms to the burden of multiple sclerosis. Adv Ther 2009; 26: 1043-1057.

3 Ginsberg D, Cruz F, Herschorn S, Gousse A, Keppenne V, Aliotta P et al. Onabotulinumtoxin $A$ is effective in patients with urinary incontinence due to neurogenic detrusor activity regardless of concomitant anticholinergic use or neurologic etiology. Adv Ther 2013; 30: 819-833.

4 Leussink VI, Husseini L, Warnke C, Broussalis E, Hartung HP, Kieseier BC. Symptomatic therapy in multiple sclerosis: the role of cannabinoids in treating spasticity. Ther Adv Neurol Disord 2012; 5: 255-266.

5 Beard S, Hunn A, Wight J. Treatments for spasticity and pain in multiple sclerosis: a systematic review. Health Technol Assess 2003; 7: 1-111.

6 http://www.ema.europa.eu/ema/index.jsp?curl=pages/medicines/human/referrals/Tetrazepam_containing_medicinal_products/human_referral_prac_000015.jsp\&mid= WCOb01ac05805c516f. Accessed August 15, 2013.

7 Sheean G. Botulinum toxin treatment of adult spasticity: a benefit-risk assessment. Drug Saf 2006; 29: 31-48.

8 Svensson J, Borg S, Nilsson P. Costs and quality of life in multiple sclerosis patients with spasticity. Acta Neurol Scand 2014; 129: 13-20.

9 Boines GJ. Predictable remissions in multiple sclerosis. Del Med J 1963; 35: 200-202

10 Müller T. Role of intraspinal steroid application in patients with multiple sclerosis. Expert Rev Neurother 2009; 9: 1279-1287.

11 Hoffmann V, Schimrigk S, Islamova S, Hellwig K, Lukas C, Brune N et al. Efficacy and safety of repeated intrathecal triamcinolone acetonide application in progressive multiple sclerosis patients. J Neurol Sci 2003; 211: 81-84.

12 Hoffmann V, Kuhn W, Schimrigk S, Islamova S, Hellwig K, Lukas C et al. Repeat intrathecal triamcinolone acetonide application is beneficial in progressive MS patients. Eur J Neurol 2006; 13: 72-76.

13 Leussink VI, Jung S, Merschdorf U, Toyka KV, Gold R. High-dose methylprednisolone therapy in multiple sclerosis induces apoptosis in peripheral blood leukocytes. Arch Neurol 2001; 58: 91-97.

14 Buttgereit F, Wehling M, Burmester GR. A new hypothesis of modular glucocorticoid actions: steroid treatment of rheumatic diseases revisited. Arthritis Rheum 1998; 41 761-767. 
15 Polman CH, Reingold SC, Banwell B, Clanet M, Cohen JA, Filippi M et al. Diagnostic criteria for multiple sclerosis: 2010 revisions to the McDonald criteria. Ann Neurol 2011; 69: 292-302.

16 Berger T. Multiple sclerosis spasticity daily management: retrospective data from Europe. Expert Rev Neurother 2013; 13 (3 Suppl 1), 3-7.

17 Tullman MJ. A review of current and emerging therapeutic strategies in multiple sclerosis. Am J Manag Care 2013; 19 (2 Suppl), S21-S27.
18 Patejdl R, Winkelmann A, Benecke R, Zettl UK. Muscle rupture caused by exacerbated spasticity in a patient with multiple sclerosis. J Neurol 2008; 255 (Suppl 6), 115-118.

19 Burks JS, Bigley GK, Hill HH. Rehabilitation challenges in multiple sclerosis. Ann Indian Acad Neurol 2009; 12: 296-306.

20 Hauser K, Walsh D. Visual analogue scales and assessment of quality of life in cancer. J Support Oncol 2008; 6: 277-282. 